\title{
Mouth Complete Response GvHD
}

National Cancer Institute

\section{Source}

National Cancer Institute. Mouth Complete Response GVHD. NCI Thesaurus. Code C126702.

An NIH modified Oral Mucosa Rating Scale (OMRS) score of 0 following previous GvHD involvement. 